British Journal of Psychiatry (1990), 157, 617-626

\title{
Correspondence
}

Editor: Ian Pullen

Contents: Neuroleptic malignant syndrome/Calciumchannel blockade and depressive illness/Investigating psychosurgery/WHO consensus statement/Benefits of routine laboratory investigations/Dyskinesia in the mentally handicapped/Linguistics of schizophrenia/Understanding Capgras syndrome/Multifactorial intoxication?/Memories in depression: pleasant or unpleasant?/Expressed emotion and lithium prophylaxis/Pseudocyesis followed by depressive psychosis/Ethics of 'brain transplants'.

\section{Neuroleptic malignant syndrome}

SIR: The wide spectrum of syndromes associated with neuroleptic chemotherapy has elicited numerous case reports documenting the neuroleptic malignant syndrome (NMS). Epidemiological arguments support the notion that case reports are subject to bias and often may render misleading or partial evidence, particularly in that we are currently lacking reliable measures to differentiate NMS from the spectrum of neuroleptic-induced extrapyramidal syndromes. In most cases of suspected NMS, the diagnosis will not be ultimately confirmed. The differential diagnosis of NMS, furthermore, presents the clinician with challenging issues. Our own clinical practice suggests that severe extrapyramidal syndromes (EPS) without fever are more common than neuroleptic-associated febrile syndromes. Patients with EPS and fever often demonstrate concurrent medical disorders. In the absence of known medical aetiology, we suggest erring on the side of caution by discontinuing neuroleptics temporarily while treating the EPS and medically evaluating the patient. The usefulness of laboratory tests, in general, lies in their role as part of extensive investigations to rule out other aetiologies of fever, altered mental status, autonomic dysfunction and extrapyramidal signs. Elevated levels of creatine phosphokinase may involve several other contributing factors such as parenteral injections, other hyperthermic syndromes, agitation, and exacerbations of psychotic episodes. Our major emphasis has been on the rapid and vigorous treatment of severe extrapyramidal rigidity with or without fever. The patient who remains agitated, and becomes diaphoretic and rigid, may develop a temperature and elevated enzyme levels as a result of untreated rigidity.

While NMS is probably more directly related to central dopamine blockade and subsequent muscle contraction, we do not recommend dantrolene sodium as a first-line treatment for suspected NMS or severe EPS. We recommend dantrolene only as an alternative when anticholinergic agents or dopamine agonists are not effective. Peet \& Collier (1990) present a case with apparent neuroleptic-induced rigidity associated with hyperthermia, change in mental status and autonomic dysfunction. There was no effort made to treat this patient vigorously with anticholinergic agents or dopamine agonists. Instead, a diagnosis of NMS was suspected without an apparent extensive medical investigation; the patient was treated with dantrolene and still remained rigid for one week. Most phenomena associated with NMS can be explained on the basis of the pharmacology of the drugs. Neuroleptics inhibit the dissipation of heat and may potentially cause disturbed hypothalamic temperature regulation. Some patients are apt to be treated with high dosages of neuroleptics, remain agitated, become diaphoretic and rigid, and develop a temperature - ergo, that is the NMS. Since we object to the term 'NMS' being used for a constellation of signs and symptoms that have not been specifically shown to be 'malignant', we urge all clinicians to understand what is really going on - namely, in most instances, a decompensated physiological state of the patient, pseudo-Parkinsonism, and agitation that is inadequately treated.

Velamoor et al (1990) report on three cases of 'incipient' NMS. There would seem to be no advantage in considering these cases as incipient forms of NMS, as this adds to the already ill-defined nature of this syndrome. Cases one and two were clearly manic. Some authors have suggested that patients with affective illness, particularly acute manic episodes with heightened metabolism, may be more 
susceptible to toxicity from neuroleptics and/or lithium (Addonizio et al, 1986; West \& Meltzer, 1979). Case one was also dehydrated with hypernatraemia a treatable concominant. Leukocytosis is a nonspecific finding that may reflect recent lithium usage, stress and/or infection. This patient remained afebrile throughout and was appropriately treated with rehydration. In addition, the patient was not reported to have rigidity in the case study, despite indications of rigidity in the discussion. It is often difficult to evaluate whether certain physiological states are secondary to NMS or specific triggering factors.

Case two had confusion with increased levels of creatine phosphokinase. His pulse, blood pressure and temperature were monitored, but no baseline values were given, and no neurological abnormality reported at the time of symptomatic treatment. Case two, the only patient with documented rigidity and fever, was treated with diazepam. This patient was a 61 -year-old male who received a total of $15 \mathrm{mg}$ of haloperidol $(750 \mathrm{mg}$ of chlorpromazine equivalents). In addition, he had cardiac disease, apparent pre-renal azotemia secondary to dehydration, electrolyte imbalance and was eventually successfully rehydrated. The physiological concomitants of ageing were not kept in mind and one may question the dosage of neuroleptic in this frail individual. We can speculate that his fever was a coincidental occurrence in a dehydrated patient. He simply had extrapyramidal side-effects that were precipitated by neuroleptics.

The main emphasis is that specific criteria and a precise delineation for the diagnosis of NMS remains controversial with some question as to the existence of a specific disorder. It would be unfortunate if preoccupation with the beguiling designation 'incipient NMS' or even the full blown phenomenon of NMS sidetracks or distorts our perception of what is really going on - that is, in most cases, the concomitants of physical illness and, most importantly neurolepticinduced rigidity possibly associated with akathisia that is inadequately treated.

The Medical College of Pennsylvania Department of Psychiatry

3200 Henry Avenue

Philadelphia

Pennsylvania 19129

\section{References}

Addonizio, G., Susman, V. L. \& Roth, S. D. (1986) Symptoms of neuroleptic malignant syndrome in 82 consecutive inpatients. American Journal of Psychiatry. 143, 1587-1590.
Peet, M. \& Collier, J. (1990) Use of carbamazepine in psychosis after neuroleptic malignant syndrome. British Journal of Psychiatry, 156, 579-581.

Velamoor, V. R., Fernando, M. L. D. \& Williamson, P. (1990) Incipient neuroleptic malignant syndrome. British Journal of Psychiatry, 156, 581-584.

West, A. P. \& MELTzer, H. Y. (1979) Paradoxical lithium neurotoxicity: a report of five cases and a hypothesis about risk for neurotoxicity. American Journal of Psychiatry, 136, 963-966.

\section{Calcium-channel blockade and depressive illness}

SIR: It was with great interest that I read Eccleston \& Cole's report of treatment-resistant depression associated with the use of a calcium-channel blocker (Journal, June 1990, 156, 889). The authors suggest that it may be possible to explain this action of dihydropyridines in terms of the monoamine hypothesis on the basis of alterations in intraneuronal concentrations of calcium. This could be mediated by dihydropyridines reducing calcium influx through voltage-sensitive calcium channels, and noradrenaline and 5-hydroxytryptamine (5-HT) altering levels of inositol 1,4,5-triphosphate (IP3) and diacylglycerol, which regulate release of sequestered calcium from intracellular organelles. However, the spatial distribution of calcium in the cell may be more important than the average cell concentration for the control of many cellular functions. For example, it has been demonstrated that transmitter release may require calcium entry through voltage-sensitive calcium channels increasing the levels of calcium just inside the membrane, rather than general cellular increases of calcium mediated by IP3 (O'Sullivan et $a l, 1989)$. The fact that dihydropyridines and monoamines can both affect intraneuronal calcium may not alone be explanation enough. Behavioural studies have clearly shown that calcium-channel blockers are able to affect $5-\mathrm{HT}_{1 \mathrm{~A}}$ and $5-\mathrm{HT}_{2}$ function in rats, effects which are reported to be independent of whole-brain 5-HT synthesis and potassium-induced release from in-vitro brain slices (Green et al, 1990). Where exactly these effects are mediated is open to debate. Since the effects of calcium-channel blockers could not be reversed by using a calcium-channel agonist (Bay K 8644), Green et al suggest that the changes in 5-HT function are not mediated at the calcium-channel level. However, only low doses of Bay K 8644 were used because of its toxicity.

Recent electrophysiological work may be able to shed light on the situation. 5-HT has been shown to be able to directly reduce calcium currents in dorsal raphe cells of rats, an effect that is independent of its previously well characterised inhibition mediated via potassium channels (Penington \& Kelly, 1990). The receptor involved appears to be the $5-\mathrm{HT}_{1 \mathrm{~A}}$ 